RDS was treated with mechanical ventilation. The MDA was higher at the neonates with the above circumstances. On the first day of life the MDA value was higher than on third day at the control. Also the MDA was significantly higher on the study group than at the control.

Conclusion The RDS at preterm is a significant risk factor for oxidative stress. The association of other diseases to RDS will increase the oxidative stress.

\section{PO-0748 NORMALISED TIDAL VOLUMES DURING HIGH FREQUENCY OSCILLATORY VENTILATION WITH THE VN-500 VENTILATOR}

1J Mazela, 'A Gradzka-Luczewska, ${ }^{2} S$ Korzan, ${ }^{2}$ S Stachnik, ${ }^{3} \mathrm{~L}$ Kramer, 'J Gadzinowski, ${ }^{4} \mathrm{M}$ Keszler. ${ }^{1}$ Neonatology, Poznan University of Medical Sciences, Poznan, Poland; ${ }^{2}$ Neonatal Research Club, Poznan University of Medical Sciences, Poznan, Poland; ${ }^{3}$ Computer Science and Statistics, Poznan University of Medical Sciences, Poznan, Poland; ${ }^{4}$ Women and Infant Hospital, Brown University, Providence, USA

\subsection{6/archdischild-2014-307384.1387}

Introduction The Babylog VN500 ventilator (Draeger, Lubeck, Germany) in High frequency oscillation mode (HFOV) has the ability to control tidal volume (VThf) using a Volume Guarantee function. However, appropriate VThf values during this mode of ventilation has not been established.

Aim The aim of this study was to establish normative data for VThf/kg during HFOV and explore its correlation with $\mathrm{FiO}_{2}$, day of life (DOL), gestational age (GA) and frequency (Freq).

Methods Newborns admitted to the level III NICU from January 2012 till September 2013 treated with VN-500 in HFOV mode according to strict clinical protocol were included. Indications for HFOV were: $\mathrm{PCO}_{2}>65$ on two consecutive blood gases and RR $>60$. Blood gases with corresponding vent settings, time, patient's weight and clinical diagnosis, were prospectively recorded. Measured VThf values were included only when $\mathrm{PCO}_{2}$ was in the 'normocapneic range' of 40-55 torr. Univariate analysis for $\mathrm{FiO}_{2}$, DOL, GA and Freq as well as Spearman's rank correlation coefficient was done.

Results 37 patients were treated with rescue HFOV; BW = $875.9 \mathrm{~g} \pm 163.7$ and $\mathrm{GA}=26.4 \pm 1.4$ (mean $\pm \mathrm{SD}$ ). 201 of 425 sets of blood gases met normocapnic criteria. The $\mathrm{PCO}_{2}$ was $46.5 \pm 3.7 \mathrm{mmHg}$ and VThf $/ \mathrm{kg} 2.00 \pm 0.59 \mathrm{~mL} / \mathrm{kg}$ (mean $\pm \mathrm{SD}$ ). Correlation with GA, DOL, $\mathrm{FiO}_{2}$ and Freq are shown in the Table (* $\mathrm{p}<0.05$ in univariate correlation).

Conclusions The mean VThf during normocapnia on HFOV is $2 \mathrm{~mL} / \mathrm{kg}$ but its value is affected by GA, DOL, $\mathrm{FiO}_{2}$ and Freq.

\begin{tabular}{lllll}
\multicolumn{4}{l}{ Abstract PO-0748 Table $\mathbf{1}$} \\
\hline Multivariate corr. & VThf/kg & VThf/kg & Spearman R & $\mathbf{p}$ \\
\hline $\mathrm{GA}($ wks): $<26$ and $>26$ & 2.02 & 1.97 & 0,162081 & 0.022 \\
$\mathrm{DOL}:<10$ and $>10$ & 1.94 & $2.15^{*}$ & 0,225533 & 0.001 \\
$\mathrm{FiO}_{2}:<0.50$ and $>0.50$ & 1.94 & $2.74^{*}$ & 0,306555 & 0.000 \\
Freq (Hz): 13 and 15 & 2.37 & $1.91^{*}$ & $-0,216868$ & 0.002 \\
\hline
\end{tabular}

\section{P0-0749 CPAP FAILURE IN VERY PRETERM INFANTS IN EUROPEAN REGIONS WITH DIFFERENT RESPIRATORY MANAGEMENT STRATEGIES: RESULTS FROM THE EPICE COHORT}

${ }^{1} \mathrm{~J}$ Mazela, ${ }^{2} \mathrm{M}$ Bonet, ${ }^{2} \mathrm{~A}$ Piedvache, ${ }^{3} \mathrm{O}$ Pryds, ${ }^{4} \mathrm{P}$ Truffert, ${ }^{5} \mathrm{PH}$ Jarreau. ${ }^{2}$. Zeitlin and the EPICE Research Group. ${ }^{1}$ Neonatology, Poznan University of Medical Sciences, Poznan,
Poland; 'Epidemiological Research Unit on Perinatal and Women's and Children's Health U953, Inserm, Paris, France; ${ }^{3}$ Neonatology, Hvidorve University Hospital, Copenhagen, Denmark; ${ }^{4}$ Medicine Neonatale, Hôpital Jeanne de Flandre, Lille, France; ${ }^{5}$ Medicine Neonatale, Hôpital Cochin-Port Royal, Paris, France

\subsection{6/archdischild-2014-307384.1388}

Background Many very preterm infants managed on early nasal continuous positive airway pressure (nCPAP) subsequently require intubation and ventilation and may suffer the consequences of delayed surfactant administration. We investigated risk factors for early nCPAP failure in European regions with diverse approaches to respiratory support.

Methods The EPICE cohort included all births between $22+0$ and $31+6$ weeks of gestation in 19 European regions in 20112012. nCPAP failure was defined as mechanical ventilation in the first $72 \mathrm{~h}$. Independent variables were gestational age, sex, multiple pregnancy, prenatal corticosteroids, pregnancy complications, small for gestational age (SGA), caesarean delivery, 5 min Apgar and region of birth. We classified regions into low $(<35 \%)$, medium $(35-55 \%)$ and high $(\geq 55 \%)$ early nCPAP use. Time to CPAP failure was modelled using Cox models.

Results Of 7566 infants admitted to neonatal care, 3360 (44\%) received early CPAP with a range from $21 \%$ to $81 \%$ across regions; $22 \%$ of infants failed CPAP, with a regional range of $11 \%$ to $61 \%$. Failure rates were $47 \%$ at $<26$ weeks, $29 \%$ at $26-$ 29 weeks and $16 \%$ at 30-31 weeks. In adjusted models, low gestational age, male sex, SGA, Apgar $<7$, no prenatal steroids, and maternal hypertension were associated with failure. Regions with low and intermediary nCPAP use had higher failure rates (adjusted hazard ratio (aHR): 1.3 95\% CI: 1.0-1.6 and aHR: $1.495 \%$ CI: $1.2-1.7$, respectively) than high-use regions.

Conclusions Perinatal factors identify infants likely to experience nCPAP failure. However, experience and training may also play an important role in effective nCPAP.

\section{PO-0750 PULMONARY HAEMORRHAGE IN PRETERM BABIES WITH RAPIDLY IMPROVING PULMONARY FUNCTION}

H Yates, L McKechnie. Department of Neonatology, Leeds Teaching Hospital NHS Trust, Leeds West Yorkshire, UK

\subsection{6/archdischild-2014-307384.1389}

Introduction Pulmonary haemorrhage affects $1-5 \%$ of babies $<32$ weeks and is associated with respiratory distress syndrome (RDS), surfactant therapy and patent ductus arteriosus (PDA). Change to our surfactant protocol was associated temporally with increased cases of pulmonary haemorrhage. We reviewed the cases for causative factors.

Methods Affected in-born babies January 1st 2012 - March 31st 2013 were included. Demographic details and clinical data pre/ post haemorrhage recorded.

Results $10 / 386$ babies $<32$ weeks affected (2.5\%). Details in Table.

All had RDS and received surfactant, 8 had repeated doses. Pulmonary haemorrhage occurred at median age of $36 \mathrm{~h}$ (IQR

\begin{tabular}{ll} 
Abstract PO-0750 Table 1 & \\
\hline Gestation, wks Mean (sd) & $27.4(2.23)$ \\
Birth weight,kg Mean (sd) & $0.96(0.22)$ \\
Maternal Steroid n & 8 \\
Surfactant n (dose mg/kg, mean, sd) & $10(284,86)$ \\
\hline
\end{tabular}

